\title{
Tuning Molecular Self-Assembly on Bulk Insulator Surfaces by Anchoring of the Organic Building Blocks
}

\author{
Philipp Rahe,* Markus Kittelmann, Julia L. Neff, Markus Nimmrich, Michael Reichling, \\ Philipp Maass, and Angelika Kühnle
}

Molecular self-assembly constitutes a versatile strategy for creating functional structures on surfaces. Tuning the subtle balance between intermolecular and molecule-surface interactions allows structure formation to be tailored at the single-molecule level. While metal surfaces usually exhibit interaction strengths in an energy range that favors molecular self-assembly, dielectric surfaces having low surface energies often lack sufficient interactions with adsorbed molecules. As a consequence, application-relevant, bulk insulating materials pose significant challenges when considering them as supporting substrates for molecular self-assembly. Here, the current status of molecular self-assembly on surfaces of wide-bandgap dielectric crystals, investigated under ultrahigh vacuum conditions at room temperature, is reviewed. To address the major issues currently limiting the applicability of molecular selfassembly principles in the case of dielectric surfaces, a systematic discussion of general strategies is provided for anchoring organic molecules to bulk insulating materials.

\section{Introduction}

Molecular self-assembly has been recognized as a powerful technique for creating functional molecular structures. ${ }^{[1]}$ When using organic molecules as building blocks, molecular self-assembly benefits from the impressive structural and functional variety of organic synthesis. ${ }^{[2]}$ Core building blocks can be equipped with a multitude of functional groups providing both, the desired functionality for a molecular device as well as suitable intermolecular interactions for controlling

Dr. P. Rahe

Department of Physics and Astronomy

115 South 1400 East

The University of Utah

Salt Lake City, UT 84112-0830, USA

E-mail: rahe@physics.utah.edu

Dr. M. Kittelmann, J. L. Neff, Dr. M. Nimmrich,

Prof. A. Kühnle

Institut für Physikalische Chemie

Duesbergweg 10-14

Johannes Gutenberg Universität Mainz

55099 Mainz, Germany

Prof. M. Reichling, Prof. P. Maass

Fachbereich Physik, Barbarastr. 7, Universität Osnabrück, 49076

Osnabrück, Germany

DOI: 10.1002/adma.201300604 the self-assembly process. To arrive at structurally well-defined arrangements, molecular self-assembly is based on reversible bond formation and breaking that allow different binding geometries to be probed to eventually reach the (local) thermodynamically stable equilibrium configuration. ${ }^{[3]}$ Especially van der Waals interactions, weak electrostatic interactions and hydrogen bonding meet the condition of reversibility at room temperature. However, while van der Waals and weak electrostatic forces exhibit an unspecific nature, the selective and directed character of hydrogen bonding has been demonstrated to enable the controlled formation of a wide range of different structures that can be steered at the single-molecule level. ${ }^{[4-8]}$ Molecular self-assembly at surfaces further benefits from the fact that structure formation is not only governed by intermolecular interactions, but also by molecule-surface interactions. In fact, the surface influence extends the variety of possible structures beyond the molecular bulk structure. Thus, tuning the subtle balance of intermolecular and molecule-surface interactions constitutes a key strategy to enrich the structural variety at the surface. ${ }^{[9,10]}$

Organic molecules typically bind more strongly to metallic than to dielectric surfaces. ${ }^{[11]}$ This strong interaction originates mainly from a hybridization of molecular orbitals with the electronic states of the metal surface, ${ }^{[12,13]}$ and generally supports surface-wetting molecular overlayers. Consequently, metal surfaces have been proven to provide a most versatile support for molecular self-assembly where in particular the influence of substrate templating can be exploited. ${ }^{[14]}$

However, to grasp the full potential of molecular selfassembly for creating application-oriented materials and devices, it is mandatory to extend the availability of selfassembly principles to dielectric surfaces. Promising application areas include surface functionalization to create for example hydrophobic, self-cleaning or biocidal coatings. Beyond surface coatings, the emerging field of molecular electronics is a major driving force when it comes to creating functional devices on a bulk insulating surface. ${ }^{[15,16]}$

Compared with metals, dielectrics constitute a rather heterogeneous class of materials spanning a wide range from weakly van der Waals bonded molecular crystals over covalent crystals such as diamond or titanium dioxide to ionic crystals such as 
potassium bromide or calcium fluoride. Thus, in the case of dielectrics, a general description of molecule-surface interactions is difficult and constitutes a significant challenge for exploring general self-assembly principles on these surfaces. As a first approach, the surface energy ${ }^{[17]}$ as a macroscopic parameter has been suggested ${ }^{[11]}$ for a classification of wetting properties.

From an experimental point of view, most surface-sensitive techniques for studying molecular self-assembly at metal surfaces such as scanning tunneling microscopy (STM), lowenergy electron diffraction (LEED) or photoelectron emission spectroscopy (PES) are electron based and, thus, require a conductive sample.

To circumvent this obstacle of experimental inaccessibility while maintaining the benefits of an insulating substrate, ultrathin insulating layers such as $\mathrm{NaCl}^{[5,18-26]} \mathrm{KBr}^{[27-30]}$ or $\mathrm{MgO}^{[31]}$ deposited on conducting substrates such as $\mathrm{Cu}(111)$, $\mathrm{Au}(111), \mathrm{Ag}(001), \mathrm{GaAs}(001)$ or $\mathrm{InSb}(001)$ have been employed as underlying substrates for subsequent molecule deposition. Based on STM imaging and spectroscopy, it has been reported by Repp and co-workers ${ }^{[24]}$ that a thin layer of $\mathrm{NaCl}$ is sufficient to largely decouple the molecular highest occupied molecular oribital (HOMO) and lowest unoccupied molecular orbital (LUMO) states from the underlying metal substrate. In their STM data, they identified the molecular orbitals of pentacene adsorbed on $\mathrm{NaCl}$ bilayers on $\mathrm{Cu}(111)$ as being largely identical to the calculated free molecule. Villagomez and coworkers ${ }^{[23]}$ have presented the direct comparison of depositing an isomer of the methylterrylene molecule on bare $\mathrm{Cu}(111)$ and on ultrathin layers of $\mathrm{NaCl}$ on $\mathrm{Cu}(111)$. Based on STM imaging and spectroscopy, they found a strong deformation of the molecular orbitals when directly deposited on the metallic $\mathrm{Cu}(111)$ surface. In sharp contrast, the HOMO and LUMO orbitals for the same molecule were directly identified when imaging the molecules on ultrathin $\mathrm{NaCl}$ layers.

This elegant principle of electronically decoupling adsorbed molecules from metallic substrates has been used in several studies to manipulate single molecules. By transferring electrons between a conducting tip and a molecule, the tautomerization, ${ }^{[32]}$ isomerization ${ }^{[33]}$ and the charge state ${ }^{[34-36]}$ of an adsorbed molecule can be changed reversibly. For example, by using a redox-reactive molecule such as bisdibenzoylmethanato-copper on $\mathrm{NaCl} / \mathrm{Cu}(111)$, the conformational and charge state change has recently been demonstrated. ${ }^{[35]}$ Interestingly, when depositing the same molecule on the bare $\mathrm{Cu}(111)$ surface, the tip-induced switching process was not observed. ${ }^{[35]}$

However, although the use of thin insulating films is a most elegant approach to electronically decouple adsorbed molecules from the underlying metal surface, thin layers are not necessarily sufficient to insulate the electronic structure of the molecules from the metal substrate. For the case of polymeric molecular wires deposited on a partly $\mathrm{NaCl}$-covered $\mathrm{Au}(111)$ surface, Bombis and co-workers ${ }^{[25]}$ estimated the tunneling leakage through the insulating film being in the same order as the electronic transport along the conjugated polymer chain. Generally, leakage currents from adsorbed structures are always expected if STM is possible on an insulating film. In this context, the usage of thick insulating layers or even bulk crystals is naturally desirable in terms of insulator-supported applicationoriented devices.

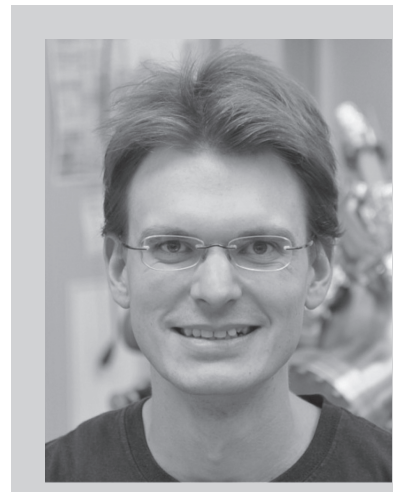

Philipp Rahe worked on his Ph.D. in the group of Angelika Kühnle at the University of Mainz and defended his thesis in 2011. Since then, he has been working as a Feodor-Lynen fellow with Clayton Williams at the University of Utah (USA), investigating the electronic structure of organic molecules.

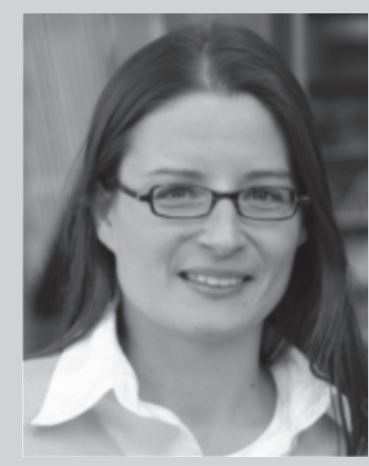

Angelika Kühnle studied physics at the Freie Universität Berlin. She joined BASF SE after her Ph.D. with Prof. Besenbacher at Aarhus University (Denmark) and established an Emmy Noether-research group at the University of Osnabrück in 2005. She was appointed as full professor in physical chemistry at the University of Mainz in 2009.

For several systems, it has been found that both, molecular diffusion and the molecular adsorption geometry differs for molecules adsorbed on the first and second insulating layer. ${ }^{[20,21,28,30]}$ Even more, the molecular adsorption geometry can differ upon deposition onto insulating thin films compared with bulk crystals of the same material. To name one example, cyano-porphyrin molecules deposited on $\mathrm{KBr}$ thin films on $\mathrm{Cu}(111)^{[27]}$ adsorb in a flat-lying orientation, but form upright-standing "nanowires" when deposited on a bulk $\mathrm{KBr}$ crystal. ${ }^{[27,37]}$ Thus, using the same molecular adsorption strategies for thin insulating films and bulk crystals does not generally yield the same results. As the deposition of molecules on bulk insulating substrates is often inevitable in terms of application-oriented device development, these examples clearly demonstrate the necessity for exploiting bulk insulating surfaces instead of the corresponding thin film systems.

For high-resolution imaging of molecular structures on bulk insulators, non-contact atomic force microscopy (NC-AFM) has been established as the ideal tool allowing for atomicresolution imaging on a routine basis. ${ }^{[38-41]}$ While atomicresolution imaging has been rather demanding in the early days of NC-AFM, constant improvements in the experimental setup ${ }^{[2,43]}$ as well as adjusting the imaging parameters ${ }^{[44]}$ have developed NC-AFM into a powerful method for atomic-scale imaging. ${ }^{41,45]}$ Nowadays, NC-AFM even allows for mapping the internal structure of single molecules. ${ }^{[46]}$

In this review, we report the current achievements of molecular self-assembly on bulk insulating surfaces investigated 

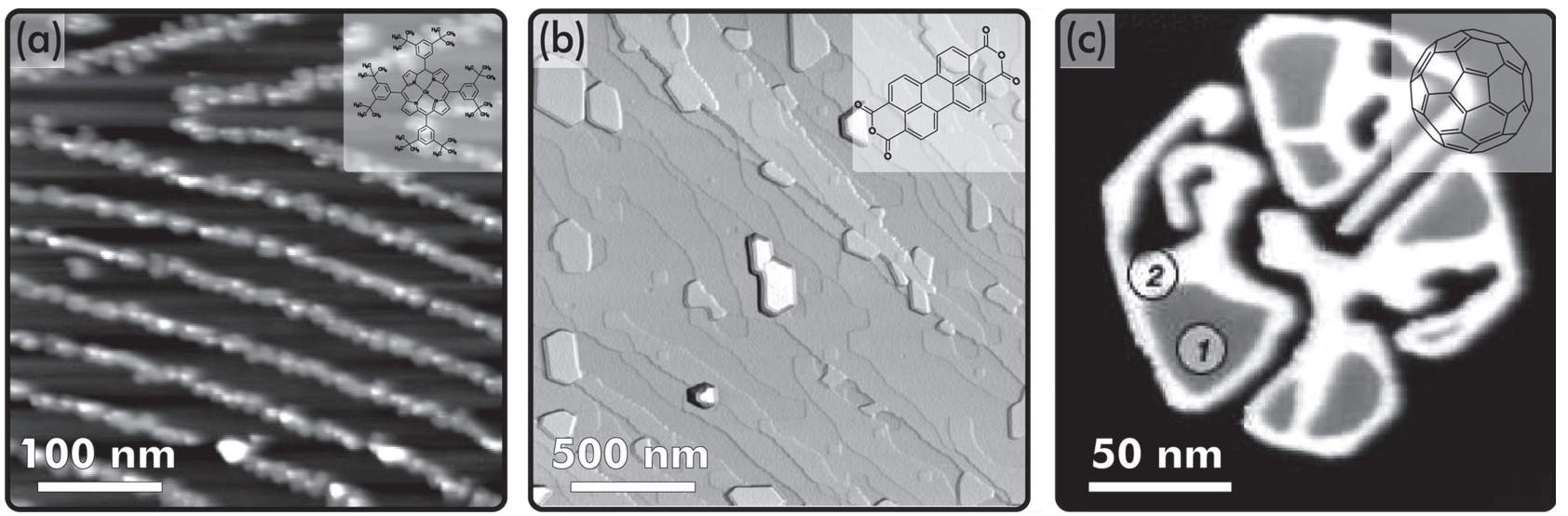

Figure 1. Pivotal experiments demonstrating weak molecule-surface interactions in the case of the prototypical insulating substrate $\mathrm{KBr}(001)$. a) $\mathrm{A}$ porphyrin derivative is shown to only decorate step edges. Reproduced with permission. ${ }^{[47]}$ Copyright 2004, IOP Publishing Ltd. b) Molecular bulk crystal formation of PTCDA at substrate step edges. Reproduced with permission. ${ }^{[51]}$ Copyright 2005, American Physical Society. c) Dewetting of $\mathrm{C}_{60}$ molecules resulting in unusual island shapes. Reproduced with permission. ${ }^{[56]}$ Copyright 2005. American Physical Society.

under ultrahigh vacuum (UHV) conditions using NC-AFM operated at room temperature. On many prototypical dielectric surfaces, the low surface energy has been shown to result in dewetting and molecular crystal formation rather than substrate templating. Thus, exploiting the full potential of molecular selfassembly on insulating surfaces requires exploring ways to increase the molecule-surface interaction. As a central theme of this review, we discuss general strategies for anchoring organic molecules to surfaces and present specific examples, illustrating the success of these general ideas on insulating substrates.

\section{Molecular Dewetting and Bulk Structure Formation on Dielectric Substrates}

One of the first in situ studies on molecular structure formation on insulating surfaces has been performed using porphyrin and perylene derivatives on $\mathrm{KBr}(001)$ and $\mathrm{Al}_{2} \mathrm{O}_{3}(0001)$ surfaces (Figure 1a). ${ }^{[47]}$ Already in this study, the weak and unspecific binding of the molecules to the surfaces has been identified as the major obstacle, limiting a simple transfer of molecular self-assembly principles from metallic to non-conducting substrates. When depositing submonolayer coverages of 3,4,9,10-perylene tetracarboxylic dianhydride (PTCDA) molecules on the $\mathrm{NaCl}(001)$ surface, monolayer islands with a $\mathrm{p}(3 \times 3)$ epitaxy have been observed. ${ }^{48]}$ This structure, however, has been shown to be metastable as a dewetting ${ }^{[49]}$ transition of the molecular film to bulk-like crystals is observed at coverages higher than $\approx 0.85$ monolayer. Another example for this so-called bimodal growth has been revealed for cobalt-salen molecules on the $\mathrm{NaCl}(001)$ surface, where metastable nanowires transform into stable nanocrystallites upon annealing the sample. ${ }^{[50]}$ Molecular dewetting has also been observed for PTCDA molecules on $\mathrm{KBr}(001)$ (Figure $1 \mathrm{~b})^{[51]}$ and $\mathrm{KCl}(001)^{[52]}$ surfaces as well as for 3,4,9,10-perylene tetracarboxylic diimide (PTCDI) molecules on $\operatorname{KBr}(001)^{[53]}$ and $\mathrm{NaCl}(001)$ substrates. ${ }^{[53,54]}$ In these studies, the high-resolution capability of NC-AFM has been exploited to confirm the weak influence of the substrate on the molecular assembly by the fact that the molecules do not adsorb in layers, but rather aggregate into a structure that closely resembles the molecular bulk crystal.

From a fundamental point of view, dewetting can be divided into two regimes. While metastable films dewet by nucleation and expansion of molecule-free regions, unstable films dewet spontaneously in a process that is referred to as spinodal decomposition. ${ }^{[55]}$ In pivotal experiments, dewetting of unstable $\mathrm{C}_{60}$ islands has been revealed on $\operatorname{KBr}(001)$ (Figure 1c) ${ }^{[56]}$ and $\mathrm{NaCl}(001)^{[57]}$ surfaces, again illustrating the low surface energy and the resulting, extremely weak molecule-surface interaction on these substrates. ${ }^{[49]}$ Interestingly, the dewetting process of $\mathrm{C}_{60}$ molecules on these surfaces results in unusual island shapes that have never been observed on metallic surfaces. Similar islands have been obtained upon deposition of $\mathrm{C}_{60}$ on another ionic surface, namely $\mathrm{CaF}_{2}(111)$, indicating the general nature of the underlying formation process. ${ }^{[58]}$ In the latter work, the origin of the unusual island morphology has been unraveled by a kinetic growth model. The island formation can be explained by the weak molecule-surface interaction, resulting in a process referred to as facilitated dewetting. Thus, while these weak interactions have been identified as a major obstacle for transferring molecular self-assembly principles from metallic to insulating substrates, the weak binding can also be the origin for novel island shapes when non-equilibrium structures are involved.

Another example, namely octadecylphosphonic acid adsorbed on mica(0001), demonstrates the influence of the preparation process on the resulting molecular structure on an insulator. ${ }^{[59]}$ Here, distinctly different molecular films (monolayers versus bilayers) have been obtained depending on the way of processing, in this case immersion into a solution instead of physical vapor deposition.

The main microscopic reason for the weak binding of organic molecules to insulating surfaces is given by the large band gap of insulating substrates. First, the presence of a large band gap causes a lack of hybridization between molecular orbitals with 
substrate surface states. ${ }^{[12,13]}$ Second, attractive van der Waals forces between insulators and adsorbed molecules are smaller as compared with metallic substrates. ${ }^{[60]}$ This is immediately evident from a direct comparison of Hamaker constants for the different material classes. ${ }^{[61-63]}$ Within the class of insulators, van der Waals forces are expected to be smaller for materials with a wider band gap as the polarizability of dielectric materials has been shown to decrease with increasing band gap. ${ }^{\text {[64] }}$ Thus, in the case of the above reviewed adsorption studies of molecules on insulating substrates, the molecules are mainly physisorbed, predominantly bonded via weak van der Waals interactions.

Wetting properties are in general controlled by the substrate/ adsorbate, substrate/vacuum and adsorbate/vacuum interfacial energies. Accordingly, for a given type of molecule, the substrate/vacuum interfacial energy, commonly referred to as the surface energy $\gamma_{\mathrm{S}}$, should be the decisive parameter. Indeed, by following the adsorption characteristics of $\mathrm{C}_{60}$ fullerenes on different substrates, this macroscopic parameter surface energy $\gamma_{\mathrm{S}}$ has been identified to give a reasonable estimate of the (de-)wetting behavior. ${ }^{[11]}$ On alkali halide surfaces with surface energies $\gamma_{S}$ in the range of 100 to $200 \mathrm{~mJ} \mathrm{~m}^{-2}$, dewetting is observed for $\mathrm{C}_{60}$ molecules. ${ }^{[57]}$ In contrast, $\mathrm{C}_{60}$ forms singlelayered islands ${ }^{[65]}$ on metal surfaces, having large surface energies of $\gamma_{\mathrm{S}}>1000 \mathrm{~mJ} \mathrm{~m}{ }^{-2}$. For insulating substrates, the calcite(104) surface constitutes an exception as, qualitatively similar to metals, single layered islands of $\mathrm{C}_{60}$ molecules are observed. ${ }^{[11]}$ The latter finding, however, is completely in line with the relatively high surface energy $\gamma_{S}$ of about $590 \mathrm{~mJ} \mathrm{~m}^{-2}$ for the calcite(104) surface. Thus, the investigated systems agree with the simple picture that molecular dewetting is favored on substrates having a low surface energy. Especially, for $\mathrm{C}_{60}$ molecules the transition from branched multilayered structures to single-layered molecular islands is found to occur between $\gamma_{\mathrm{S}} \approx 480 \mathrm{~mJ} \mathrm{~m}{ }^{-2}$ (on $\mathrm{CaF}_{2}(111)$ ) and $\gamma_{\mathrm{S}} \approx 590 \mathrm{~mJ} \mathrm{~m} \mathrm{~m}^{-2}$ (on calcite(104)). A plot depicting various surface energies $\gamma_{S}$ of metallic and ionic materials, surface energies $\gamma_{\mathrm{m}}$ of molecular crystals as well as the impact of specific combinations on molecular dewetting is given in Figure 2.

\section{From Bulk Structure Formation to Substrate Templating}

Soon after initial experiments demonstrated the weak influence of insulating surfaces on molecular self-assembly, strategies have been explored to enhance not only the templating effect of the surface on the molecular structure formation, but specifically the creation of nucleation centers for the molecular film. In a first attempt to realize this strategy, the underlying surface has been patterned to guide the molecule nucleation at step edges and surface pits. ${ }^{[37,66-71]}$ By irradiation with an electron beam, monolayer deep pits can be produced on $\operatorname{KBr}(001),{ }^{[72]}$ which can provide step edges acting as nucleation sites for deposited molecules ${ }^{[37]}$ (Figure 3a) or can even trap complete molecular islands. ${ }^{[71]}$

Another possibility for the creation of nucleation centers is the deposition of a bonding agent, featuring a strong bond to the surface as well as to adsorbed molecules. Ideally, this bonding agent should be designed not to interfere with the

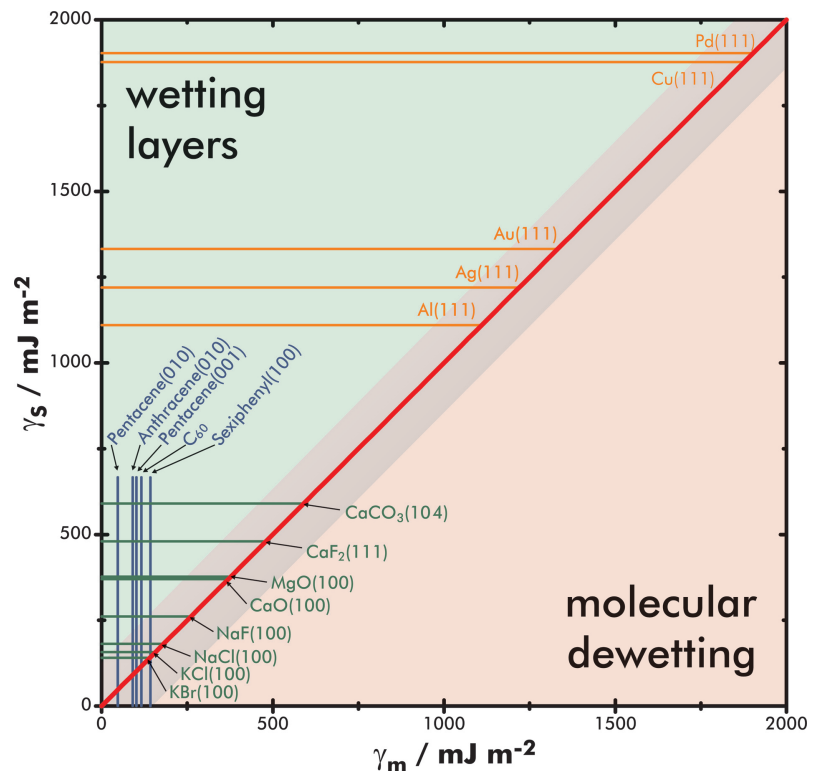

Figure 2. Comparison of surface energies $\gamma_{\mathrm{s}}$ of various dielectric and metallic substrates with surface energies $\gamma_{m}$ of molecular crystals. According to well-established growth theory, ${ }^{[17]}$ combinations of these energies yielding molecular wetting is expected in the upper-left green shaded area. ${ }^{[1]}$ The red diagonal line depicts the transition between these two regimes for the case of zero interface energy.

molecular functionality. One example is the deposition of a metal such as Au clusters on $\operatorname{KBr}(001) \cdot{ }^{[75]}$ In this case, the metal clusters act as nucleation centers steering the growth of subsequently deposited porphyrin molecules towards the formation of molecular wires, interconnecting two clusters.

As a third strategy, defects in or close to the surface can be utilized for forming a strong bond with few deposited molecules and, thus, act as a nucleation center. ${ }^{[76]}$

For anchoring single molecules and molecular structures to dielectric surfaces, different strategies have been pursued over the last years. On ionic crystals, exploiting electrostatic interactions emerges naturally as a promising approach for anchoring molecules to the crystal surfaces. To enhance the electrostatic molecule-surface interaction, the intermolecular charge distribution should match the electrostatic potential periodicity of the ionic crystal lattice. This strategy has been followed for the formation of up to $600 \mathrm{~nm}$ long molecular wires made of a perylene derivative on $\operatorname{KBr}(001)$ (Figure 3b). ${ }^{[73,77]}$ For the adsorption of cobalt-salen molecules on $\mathrm{NaCl}(001)$ surfaces, a pure electrostatic interaction between the cobalt metal atom and the surface chloride anions has been found. ${ }^{[78]}$ The adsorption energy has been calculated by means of DFT to be of the order of 0.6 to $0.7 \mathrm{eV}^{[50]}$ Along this line, molecules bearing a large dipole moment have been explored for increasing the electrostatic interaction with the surface. ${ }^{[37,69,74,79-81]}$ For chloro-[subphthalocyaninato]-boron-(III) (SubPc) molecules on nanostructured $\mathrm{KBr}(001)$ surfaces, it has been shown that the molecules prefer step edges as adsorption sites. ${ }^{[69]}$

By benefiting from the flexibility of chemical synthesis, molecules can be tailored by attaching specifically designed end groups. For instance, non-polar molecules can be equipped 

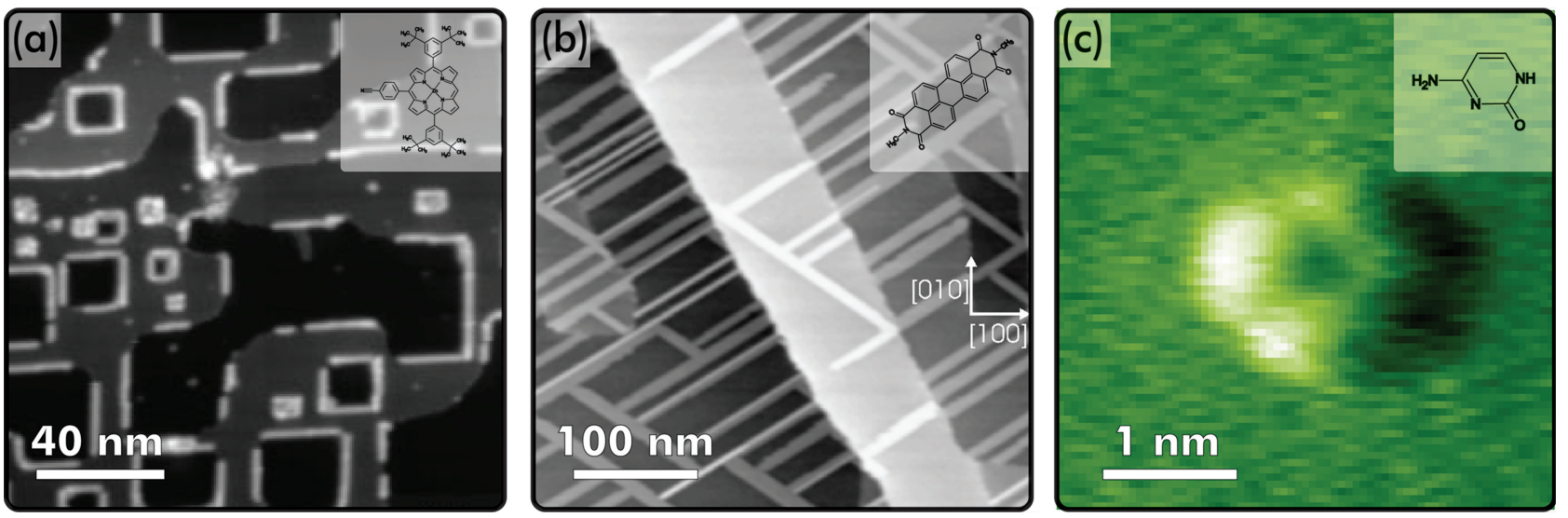

Figure 3. Increasing surface templating. a) Upon electron irradiation, surface pits are created on $\mathrm{KBr}(001)$ that act as molecule nucleation sites. Reproduced with permission. ${ }^{[37]}$ Copyright 2008, John Wiley and Sons. b) The charge distribution within a perylene derivative results in electrostatic interaction with the underlying $\mathrm{KBr}(001)$ surface. Reproduced with permission. ${ }^{[73]}$ Copyright 2007, American Institute of Physics. c) Cooperative mechanism for anchoring cytosine trimers onto $\mathrm{CaF}_{2}(111)$. Reproduced with permission. ${ }^{[74]}$ Copyright 2009, American Physical Society.

with polar moieties. Usually, when adsorbed on ionic crystals, molecules are observed to bind electrostatically to the substrate's step edges such as in the case of cyanoporphyrins on $\operatorname{KBr}(001) \cdot{ }^{[37]}$ Two different kinds of ordered overlayers have been observed for a triphenylene derivative on $\operatorname{KBr}(001)$ surfaces. ${ }^{[82]}$ In this study, six cyano groups have been attached to the triphenylene core using flexible propyl chains that are intended to guarantee optimum matching with the substrate lattice. Indeed, for flat-lying molecules an adsorption energy as high as $1.8 \mathrm{eV}$ has been estimated. Due to the flexibility of this comparatively large molecule, however, the diffusion barrier has been identified to be considerably lower than the adsorption energy, resulting in rapid diffusion at room temperature. Moreover, the overlayer composed of flat-lying molecules has been observed to undergo dewetting over time, resulting in a more stable structure of upright-standing molecules. The reorganized molecules are stabilized by $\pi-\pi$ stacking with an intermolecular binding energy of the order of $2.5 \mathrm{eV}$. For truxene molecules, specifically functionalized with three benzonitrile groups to yield a dipole moment of $\approx 4.5 \mathrm{D}$, the increased molecule-substrate interaction resulted in anchoring to substrate step edges. ${ }^{[81]}$ However, even anchored molecules have been observed to perform rapid movements along the step edges due to a very low diffusion barrier. A rough estimation for the diffusion barrier in the latter study yields about $0.3 \mathrm{eV},{ }^{[83]}$ facilitating diffusion at room temperature.

To increase the molecule-substrate interaction, a cooperative mechanism of molecule anchoring has been identified for cytosine on $\mathrm{CaF}_{2}$ (111) (Figure 3c). ${ }^{[74]}$ In this example, the electric dipole moment of the individual molecules has a value of 6 to $8 \mathrm{D}$ and fits excellently in size to the distance of the $\mathrm{CaF}_{2}(111)$ surface ions, but individual molecules experience a diffusion barrier of approximately $0.5 \mathrm{eV}$, which is easily overcome at room temperature. However, besides the electrostatic interaction with the surface, cytosine molecules can form intermolecular hydrogen bonds. When three-membered rings are formed, the individual molecules within the ring can adopt the same adsorption position as a single molecule due to the three-fold symmetry of the underlying lattice. Therefore, each molecule can attain the optimum adsorption geometry with respect to the substrate. In such three-membered rings, a simple summation of the individual diffusion barriers can serve as a rough estimate for the diffusion barrier of the entire cluster, resulting in a barrier of approximately $1.5 \mathrm{eV}$. This barrier is sufficiently large to immobilize the rings at room temperature. Consequently, the increased molecule-surface interaction of individual molecules together with the arrangement in three-membered hydrogen-bonded rings eventually results in the immobilization of trimer clusters on the surface. It is important to note, however, that only the cooperative combination of these two interactions limits the mobility of the cytosine molecules at room temperature. Another example demonstrating the stabilization by a combination of different interaction types are racemic heptahelicene-2-carboxylic acid ([7]HCA) molecules deposited on calcite(104). ${ }^{[84]}$ When deposited at room temperature, the molecules assemble to molecular rows of well-defined width. As has been shown from the deposition of a pure [7]HCA enantiomer, ${ }^{[85]}$ the rows are composed of racemic molecular pairs linked by hydrogen bonds. The molecular rows perfectly align along the [010] surface direction and are stabilized by $\pi-\pi$ interactions between the molecular pairs. It has, furthermore, been suggested that the molecules adapt a slightly tilted orientation to match the substrate periodicity, thus presenting a templating effect of the underlying substrate.

Upon studying the adsorption of a zwitterionic stilbazolium derivative with a very high dipole moment $(\approx 16.8 \mathrm{D})$ as a function of the substrate material $(\mathrm{NaCl}, \mathrm{KCl}, \mathrm{RbCl}$, and $\mathrm{KBr})$, the impact of changing the substrate lattice periodicity has been investigated. ${ }^{[80]} \mathrm{A}$ Moiré pattern has been observed for some of the substrates, indicating that the respective substrate surface significantly affects the structure formation.

The stabilizing effect of intermolecular hydrogen bonds has, furthermore, been exploited in a recent study utilizing the unusually strong intermolecular cohesion energy of boronic acid to stabilize a wetting layer on $\mathrm{KCl}(001)$ at room tempera- 
ture. ${ }^{[86]}$ In this study, the substrate has been shown to have a marginal influence on the resulting molecular film, illustrating that the strong intermolecular cohesion rather than the molecule-surface adhesion is responsible for the stabilization of the film.

From these examples, it is evident that a stronger molecule-substrate interaction is mandatory for tuning molecular self-assembly that goes beyond dewetting and bulk structure formation. The above mentioned transition from a thermodynamically unstable layer of flat-lying triphenylene derivatives on $\mathrm{KBr}(001)$ into a cohesion-driven film readily emphasizes the need for increasing the influence of the substrate. This has recently been demonstrated by the coexistence of a bulk-like structure and a substrate-templated phase. ${ }^{[87]} \mathrm{A}$ delicate balance between molecular bulk crystal formation and substrate templating has been revealed by the adsorption structure of biphenyl-4,4'-dicarboxylic acid (BPDCA) on calcite(104). ${ }^{[87]}$ This molecule forms two coexisting structures at room temperature, indicating that both structures are energetically very similar. One of the structures closely resembles the hydrogen-bonded configuration of the molecular bulk crystal, demonstrating a marginal influence of the underlying substrate. The second structure, however, is found to be clearly dictated by the interaction of the carboxylic acid groups with the substrate carbonate groups. The formation of this structure is driven by the excellent size match of the distance between the carboxylic acid groups at each molecule to the substrate carbonate group spacing, resulting in an increased binding towards the surface and, therefore, to a strong templating effect.

\section{Strategies for Anchoring Organic Molecules to a Dielectric Surface at Room Temperature}

Several general strategies can be discussed for anchoring organic molecules to dielectric surfaces at room temperature. Here we identify three main aspects and illustrate these by specific examples. To keep the concepts as general as possible, however, we do not consider the creation of specific nucleation sites on the surface such as step edges or surface defects in the presented strategies.

\subsection{Interaction Type and Strength}

The interaction with the surface is controlled by the type and strength of the molecule-surface binding (Table 1, Figure 4a). Besides the strength and the range of the binding, the specific nature of the interactions constitutes the key controlling parameter for molecular self-assembly as it steers, for instance, the orientation of the molecules with respect to each other and with respect to the substrate. Strong bonds with a covalent character are usually absent in molecule-insulator systems. Van der Waals interaction forces are typically weak and unspecific, while hydrogen bonds are very specific and directed; this is why the latter frequently play a crucial role in molecular self-assembly. ${ }^{[4-8]}$

Dielectric surfaces can allow for hydrogen bond formation between adsorbed molecules and the substrate surface. For
Table 1. Molecule-surface interaction types, their typical interaction strength and range.

\begin{tabular}{lccc}
\hline Bond type & $\begin{array}{c}\text { Typical interaction } \\
\text { strength }\end{array}$ & $\begin{array}{c}\text { Typical interaction } \\
\text { range }\end{array}$ & Bond characteristics \\
\hline van der Waals & $\approx 0.02-0.1 \mathrm{eV}$ & $<1 \mathrm{~nm}$ & unspecific \\
hydrogen bonds & $\approx 0.1-0.6 \mathrm{eV}$ & $\approx 0.25 \mathrm{~nm}$ & specific and directional \\
electrostatic/ionic & $\approx 0.1-2.5 \mathrm{eV}$ & long-range & unspecific \\
\hline
\end{tabular}

example, a theoretical study has elucidated the case of salicylic acid adsorbed on the $\mathrm{MgSO}_{4} \cdot \mathrm{H}_{2} \mathrm{O}(100)$ surface. In this system, a hydrogen bond is formed between the phenolic hydroxyl group of the molecule and a sulfate ion of the surface. ${ }^{[8]}$ On the same surface, hydrogen bonds play an even more decisive role in the stabilization of water molecules by forming two bridges to each water molecule. Thereby, the water molecule is fixed on the surface, with the oxygen atom located above the magnesium ion of the surface. ${ }^{[8]}$ The formation of hydrogen bonds between adsorbed water molecules and dielectric surfaces has also been predicted theoretically for the case of $\mathrm{MgO}(001) \cdot{ }^{[90]}$ As an experimental example, calcite(104) can act as a proton acceptor, thus providing very specific anchoring sites for proton donor molecules, such as carboxylic acid groups. ${ }^{[4,87]}$ It has, however, to be noted that the formation of a single hydrogen bond with typical interaction strengths of the order of 0.1 to $0.3 \mathrm{eV}$ is insufficient for stable anchoring of molecules at room temperature. The same argument holds for the electrostatic interaction of molecules bearing a large dipole moment with an ionic substrate surface. Although this strategy has been identified as a promising strategy for anchoring organic molecules to surfaces, ${ }^{[74,80,81]}$ again the binding of organic mol-

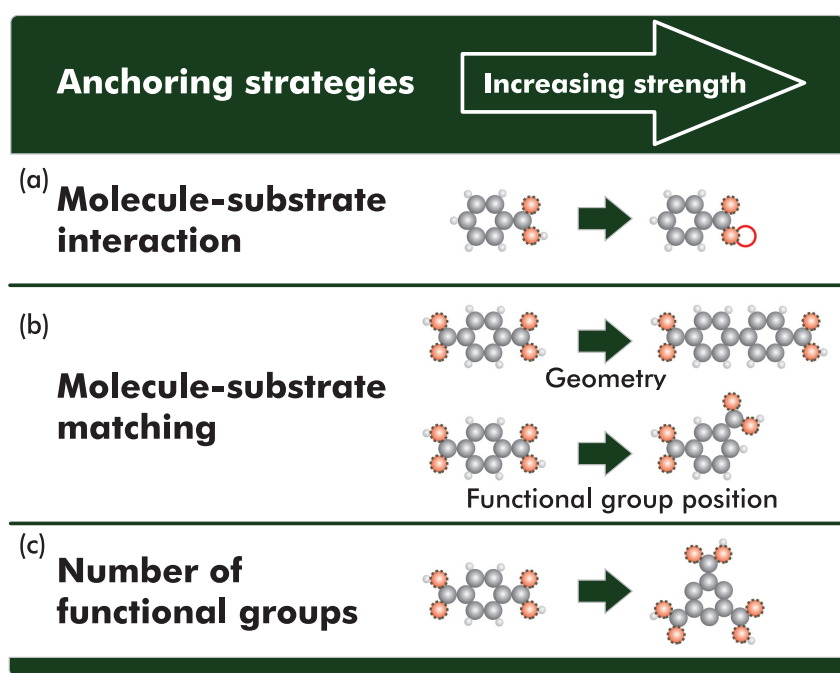

Figure 4. Strategies for molecular anchoring. a) Increasing the strength and character of the individual bonds. b) Matching the molecular structure to the surface periodicity achieved by changing the molecular core or by changing the position of anchoring groups at the molecular core. c) Increasing the number of anchoring groups to increase the binding to the surface. 


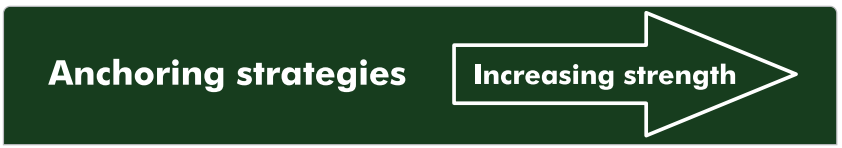

(a) Molecule-substrate interaction
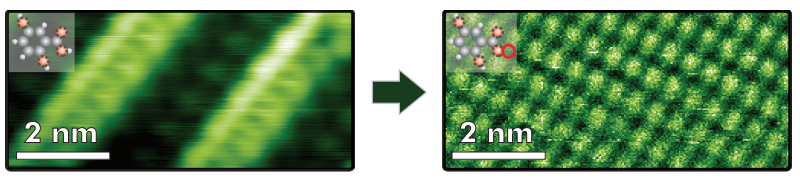

(b)

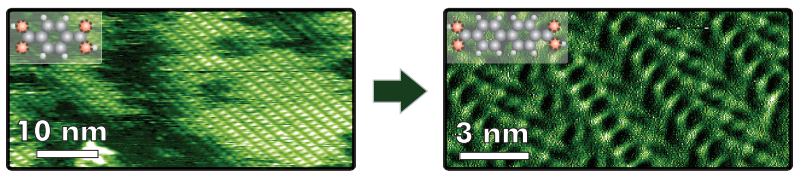

(c)

Number of functional groups

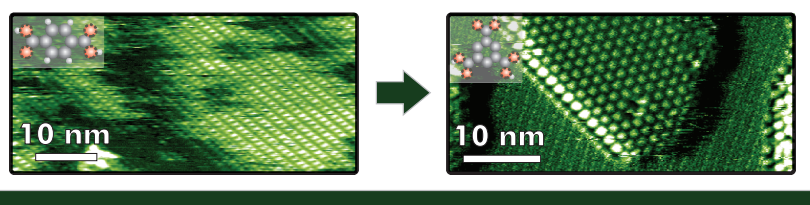

Figure 5. Realization of anchoring strategies on calcite(104). a) The binding to the substrate is increased by deprotonating DHBA molecules. Reproduced with permission. ${ }^{[91]}$ Copyright 2012, American Chemical Society. b) By adding a second benzene ring to a TPA molecule, yielding BPDCA, the molecules align in a more favorable adsorption position. Left image: Reproduced with permission. ${ }^{[4]}$ Copyright 2012, John Wiley and Sons. Right image: Reproduced with Permission. ${ }^{[87]}$ Copyright 2012, IOP Publishing Ltd. c) The change from two (in TPA) to three (in TMA) carboxylic acid endgroups leads to a significantly more stable molecular structure. Reproduced with permission. ${ }^{[4]}$ Copyright 2012, John Wiley and Sons.

ecules based on dipole interactions solely is typically insufficient to firmly anchor the molecules to a bare terrace at room temperature $^{[81]}$ if another cooperative effect such as additional intermolecular cohesion based on hydrogen bonding, ${ }^{[74]}$ the formation of an extended molecular layer ${ }^{[80]}$ or the presence of numerous highly polar endgroups ${ }^{[9]}$ are absent. Recently, the interaction of 2,5-dihydroxybenzoic acid with calcite(104) has been increased by changing the interaction type upon molecule deprotonation. ${ }^{[91]}$ At room temperature, the molecules undergo a transition from the protonated carboxylic acid to the deprotonated carboxylate state. Accordingly, the interaction with the surface changes from weak hydrogen bonding in the protonated state to electrostatic interaction in the deprotonated state (Figure 5a). As dielectric surfaces can be very different in nature, it is mandatory to consider the possible interaction characteristics with organic molecules and tailor their functional groups accordingly.

\subsection{Molecule-Substrate Matching}

An important aspect in molecule-surface anchoring is matching the molecular structure to the substrate periodicity, as has been indicated by the comparison of the adsorption structure of a stilbazolium derivative on different insulating surfaces. ${ }^{[80]}$ Considering a given insulating material as supporting substrate, the complementary strategy is to adjust the molecular building blocks to the substrate of interest. Two approaches can be followed in this case (Figure 4b). First, the molecular core can be adjusted to match the substrate periodicity. An example is given by comparing the adsorption structure of benzene-1,4-dicarboxylic acid (terephthalic acid) (TPA) ${ }^{[4]}$ and $\mathrm{BPDCA}^{[87]}$ on calcite(104) (Figure 5b). In both cases, the interaction of the protonated molecule with the substrate is presumably governed by the interaction of the carbonyl oxygen atom with the surface calcium cation, while the hydrogen atom forms a hydrogen bond with the protruding oxygen atom of the surface carbonate group. Both molecules are equipped with two carboxylic acid groups in para position, and, hence, the number and position of the functional groups are identical. However, while the TPA core is constituted by a single benzene ring, BPDCA consists of a biphenyl core, obviously resulting in a larger spacing of the carboxylic acid groups. Although TPA forms a well-ordered $(2 \times 2)$ superstructure, these islands are highly mobile at sub-monolayer coverages. In contrast, the BPDCA superstructure seems to fit better in size to the underlying substrate, specifically both carboxylic acid groups apparently adopt more favorable adsorption sites compared with TPA. Consequently, these experiments suggest that the BPDCA structure is indeed anchored more firmly to the surface as is conferred from the more stable edges of the BPDCA islands.

The second approach for adjusting the molecular building block to the substrate is to change the position of the anchor group at the molecular core to better match the substrate structure (Figure 5b).

\subsection{Number of Functional Groups}

An obvious concept for increasing the influence of the surface is increasing the number of functional groups that provide an interaction with the surface (Figure 4c). This approach has been the driving force of many attempts in literature, including the above mentioned functionalization of truxene with benzonitrile groups $^{[81]}$ and the functionalization of a triphenylene core with six flexible propyl chains ending with polar cyano groups. ${ }^{[79]}$ In the latter study, the calculated adsorption energy is as large as $1.8 \mathrm{eV}$, mainly due to the ionic binding of the six cyano groups to the substrate. Another example is changing the number of carboxylic acid groups at a benzene core from two as in TPA to three as in benzene-1,3,5-tricarboxylic acid (trimesic acid, TMA) (Figure 5c). ${ }^{[4]}$ In the close-packed island (the so-called flower motif), the TMA molecules adopt equivalent adsorption positions in a $c(2 \times 2)$ superstructure. In this configuration, all three carboxylic acid groups can interact with the substrate, resulting in a more stable configuration as compared with the TPA islands. It is important to note that the TMA structure is slightly compressed in this configuration as compared with the perfectly hexagonal flower motif, clearly demonstrating the templating influence of the substrate. The resulting island stability is likely a cooperative effect of the molecular cohesion and 
the increased binding to the underlying substrate due to a third functional group.

\section{Conclusions}

Molecular self-assembly at surfaces is a most powerful tool for creating functional materials in a spontaneous, parallel fashion. However, a simple transfer of the formation principles from metallic to application-relevant dielectric surfaces is not straightforward due to the diverse character of dielectric materials. Prototypical insulating substrates studied so far have posed significant challenges for molecular self-assembly studies mainly due to the weak and unspecific interaction with the organic building blocks. This weak interaction is known to result in molecular bulk crystal growth without the possibility of tailoring the structure. Thus, although the weak interaction opens up conceptually new strategies of structure formation based on transient non-equilibrium structures, it severely limits molecular self-assembly when considering thermodynamically stable structures. Consequently, increasing the molecule-surface interaction has been in the focus recently, resulting in a growing number of molecular structures that can be controlled at the single-molecule level. Successful examples of molecular self-assembly on dielectric surfaces are based on tailoring the interaction type, the detailed moleculesurface matching and the number of anchoring points per molecule. Thus, despite the heterogeneous nature of dielectric materials spanning from covalent to van der Waals crystals, general strategies for anchoring organic molecules to dielectric surfaces can be identified. Understanding the underlying principles provides the basis for developing molecular selfassembly on insulating surfaces into an application-oriented framework.

\section{Acknowledgements}

This work was supported by the German Research Foundation (DFG) through the SFB 625, TP B17. P.R. gratefully acknowledges financial support from the Alexander von Humboldt Foundation.

Received: February 5, 2013

Revised: March 25, 2013

Published online: June 12, 2013

[1] J. M. Lehn, Angew. Chem. 1988, 100, 91.

[2] B. Lucas, T. Trigaud, C. Videlot-Ackermann, Polym. Int. 2012, 61, 374.

[3] G. M. Whitesides, J. P. Mathias, C. T. Seto, Science 1991, 254, 1312.

[4] P. Rahe, M. Nimmrich, A. Kühnle, Small 2012, 8, 2968.

[5] C. Bombis, N. Kalashnyk, W. Xu, E. Lægsgaard, F. Besenbacher, T. R. Linderoth, Small 2009, 5, 2177.

[6] W. Xu, M. Dong, H. Gersen, E. Rauls, S. Vazquez-Campos, M. Crego-Calama, D. N. Reinhoudt, I. Stensgaard, E. Laegsgaard, T. R. Linderoth, F. Besenbacher, Small 2007, 3, 854.

[7] S. De Feyter, A. Miura, S. Yao, Z. Chen, F. Würthner, P. Jonkheijm, A. Schenning, E. W. Meijer, F. C. De Schryver, Nano Lett. 2005, 5, 77.

[8] C. T. Seto, G. M. Whitesides, J. Am. Chem. Soc. 1991, 113, 712.
[9] M. Einax, W. Dieterich, P. Maass, Rev. Mod. Phys. 2013, in press.

[10] A. Kühnle, Curr. Opin. Colloid Interface Sci. 2009, 14, 157.

[11] P. Rahe, R. Lindner, M. Kittelmann, M. Nimmrich, A. Kühnle, Phys. Chem. Chem. Phys. 2012, 14, 6544.

[12] A. Hauschild, K. Karki, B. C. C. Cowie, M. Rohlfing, F. S. Tautz, M. Sokolowski, Phys. Rev. Lett. 2005, 94, 036106.

[13] J. Ziroff, F. Forster, A. Schoell, P. Puschnig, F. Reinert, Phys. Rev. Lett. 2010, 104, 233004.

[14] J. V. Barth, Annu. Rev. Phys. Chem. 2007, 58, 375.

[15] C. Joachim, M. A. Ratner, Proc. Natl. Acad. Sci. USA 2005, 102, 8801.

[16] C. Joachim, J. K. Gimzewski, A. Aviram, Nature 2000, 408, 541.

[17] E. Bauer, Z. Kristallogr. 1958, 110, 372.

[18] M. Abel, S. Clair, O. Ourdjini, M. Mossoyan, L. Porte, J. Am. Chem. Soc. 2011, 133, 1203.

[19] L. Gross, R. R. Schlittler, G. Meyer, L. A. Fendt, F. Diederich, T. Glatzel, S. Kawai, S. Koch, E. Meyer, J. Vac. Sci. Technol. B 2010, 28, C4D34.

[20] L. Ramoino, M. von Arx, S. Schintke, A. Baratoff, H. J. Guentherodt, T. A. Jung, Chem. Phys. Lett. 2006, 417, 22.

[21] H. Karacuban, S. Koch, M. Fendrich, T. Wagner, R. Möller, Nanotechnology 2011, 22, 295305.

[22] E. Le Moal, M. Müller, O. Bauer, M. Sokolowski, Phys. Rev. B 2010, $82,045301$.

[23] C. J. Villagomez, T. Zambelli, S. Gauthier, A. Gourdon, S. Stojkovic, C. Joachim, Surf. Sci. 2009, 603, 1526.

[24] J. Repp, G. Meyer, S. M. Stojkovic, A. Gourdon, C. Joachim, Phys. Rev. Lett. 2005, 94, 026803.

[25] C. Bombis, F. Ample, L. Lafferentz, H. Yu, S. Hecht, C. Joachim, L. Grill, Angew. Chem. Int. Ed. 2009, 48, 9966.

[26] F. Rossel, M. Pivetta, F. Patthey, E. Cavar, A. P. Seitsonen, W.-D. Schneider, Phys. Rev. B 2011, 84, 075426.

[27] T. Glatzel, L. Zimmerli, S. Kawai, E. Meyer, L.-A. Fendt, F. Diederich, Beilstein J. Nanotechnol. 2011, 2, 34.

[28] C. Loppacher, U. Zerweck, L. M. Eng, S. Gemming, G. Seifert, C. Olbrich, K. Morawetz, M. Schreiber, Nanotechnology 2006, 17, 1568.

[29] U. Zerweck, C. Loppacher, L. M. Eng, Nanotechnology 2006, 17, S107.

[30] B. Such, G. Goryl, S. Godlewski, J. J. Kolodziej, M. Szymonski, Nanotechnology 2008, 19, 475705.

[31] S. Schintke, S. Messerli, M. Pivetta, F. Patthey, L. Libioulle, M. Stengel, A. De Vita, W.-D. Schneider, Phys. Rev. Lett. 2001, 87, 276801.

[32] P. Liljeroth, J. Repp, G. Meyer, Science 2007, 317, 1203.

[33] A. Safiei, J. Henzl, K. Morgenstern, Phys. Rev. Lett. 2010, 104, 216102.

[34] S. W. Wu, N. Ogawa, G. V. Nazin, W. Ho, J. Phys. Chem. C 2008, $112,5241$.

[35] T. Leoni, O. Guillermet, H. Walch, V. Langlais, A. Scheuermann, J. Bonvoisin, S. Gauthier, Phys. Rev. Lett. 2011, 106, 216103.

[36] I. Swart, T. Sonnleitner, J. Repp, Nano Lett. 2011, 11, 1580.

[37] S. Maier, L.-A. Fendt, L. Zimmerli, T. Glatzel, O. Pfeiffer, F. Diederich, E. Meyer, Small 2008, 4, 1115.

[38] F. J. Giessibl, Science 1995, 267, 68.

[39] L. Gross, Nat. Chem. 2011, 3, 273.

[40] F. J. Giessibl, Rev. Modern Phys. 2003, 75, 949.

[41] C. Barth, A. S. Foster, C. R. Henry, A. L. Shluger, Adv. Mater. 2011, 23, 477.

[42] S. Torbrügge, J. Lübbe, L. Tröger, M. Cranney, T. Eguchi, Y. Hasegawa, M. Reichling, Rev. Sci. Instrum. 2008, 79, 083701.

[43] J. Lübbe, M. Temmen, S. Rode, P. Rahe, A. Kühnle, M. Reichling, Beilstein J. Nanotechnol. 2012, 4, 32.

[44] F. J. Giessibl, S. Hembacher, M. Herz, C. Schiller, J. Mannhart, Nanotechnology 2004, 15, S79.

[45] M. Reichling, C. Barth, Phys. Rev. Lett. 1999, 83, 768. 
[46] L. Gross, F. Mohn, N. Moll, P. Liljeroth, G. Meyer, Science 2009, 325, 1110.

[47] L. Nony, R. Bennewitz, O. Pfeiffer, E. Gnecco, A. Baratoff, E. Meyer, T. Eguchi, A. Gourdon, C. Joachim, Nanotechnology 2004, 15, S91.

[48] S. A. Burke, W. Ji, J. M. Mativetsky, J. M. Topple, S. Fostner, H. J. Gao, H. Guo, P. Grütter, Phys. Rev. Lett. 2008, 100, 186104.

[49] S. A. Burke, J. M. Topple, P. Grütter, J. Phys.: Condens. Matter 2009, $21,423101$.

[50] S. Fremy, A. Schwarz, K. Lämmle, M. Prosenc, R. Wiesendanger, Nanotechnology 2009, 20, 405608.

[51] T. Kunstmann, A. Schlarb, M. Fendrich, T. Wagner, R. Möller, R. Hoffmann, Phys. Rev. B 2005, 71, 121403.

[52] T. Dienel, C. Loppacher, S. C. B. Mannsfeld, R. Forker, T. Fritz, Adv. Mater. 2008, 20, 959.

[53] J. M. Topple, S. A. Burke, W. Ji, S. Fostner, A. Tekiel, P. Grütter, J. Phys. Chem. C 2011, 115, 217.

[54] J. M. Topple, S. A. Burke, S. Fostner, P. Grütter, Phys. Rev. B 2009, 79, 205414.

[55] P.-G. de Gennes, F. Brochard-Wyart, D. Quéré, Capillarity and Wetting Phenomena, Springer Verlag, New York 2004.

[56] S. A. Burke, J. M. Mativetsky, R. Hoffmann, P. Grütter, Phys. Rev. Lett. 2005, 94, 096102

[57] S. A. Burke, J. M. Mativetsky, S. Fostner, P. Grütter, Phys. Rev. B 2007, 76, 035419

[58] M. Körner, F. Loske, M. Einax, A. Kühnle, M. Reichling, P. Maass, Phys. Rev. Lett. 2011, 107, 016101.

[59] C. Loppacher, U. Zerweck, D. Koehler, M. Rodenstein, E. Jaehne, R. Luther, H.-J. Adler, L. M. Eng, Nanotechnology 2007, 18, 084003.

[60] J. N. Israelachvili, Intermolecular and Surface Forces, 3rd ed., Academic Press, London, UK 2011.

[61] L. Bergström, Adv. Colloid Interface Sci. 1997, 70, 125.

[62] D. B. Hough, L. R. White, Adv. Colloid Interface Sci. 1980, 14, 3.

[63] V. A. Parsegian, G. H. Weiss, J. Colloid Interface Sci. 1981, 81, 285.

[64] N. M. Ravindra, P. Ganapathy, J. Choi, Infrared Phys. Technol. 2007, $50,21$.

[65] T. David, J. K. Gimzewski, D. Purdie, B. Reihl, R. R. Schlittler, Phys. Rev. B 1994, 50, 5810 .

[66] J. M. Mativetsky, S. A. Burke, S. Fostner, P. Grütter, Small 2007, 3, 818.

[67] S. A. Burke, J. M. LeDue, J. M. Topple, S. Fostner, P. Grütter, Adv. Mater. 2009, 21, 2029.

[68] O. H. Pakarinen, J. M. Mativetsky, A. Gulans, M. J. Puska, A. S. Foster, P. Grütter, Phys. Rev. B 2009, 80, 085401.

[69] L. Nony, E. Gnecco, A. Baratoff, A. Alkauskas, R. Bennewitz, O. Pfeiffer, S. Maier, A. Wetzel, E. Meyer, C. Gerber, Nano Lett. 2004, $4,2185$.
[70] L. Zimmerli, S. Maier, T. Glatzel, E. Gnecco, O. Pfeiffer, F. Diederich, L. Fendt, E. Meyer, J. Phys.: Conf. Ser. 2007, 61, 1357.

[71] J. M. Mativetsky, S. A. Burke, S. Fostner, P. Grütter, Nanotechnology 2007, 18, 105303.

[72] R. Bennewitz, S. Schär, V. Barwich, O. Pfeiffer, E. Meyer, F. Krok, B. Such, J. Kolodzej, M. Szymonski, Surf. Sci. 2001, 474, L197.

[73] M. Fendrich, T. Kunstmann, Appl. Phys. Lett. 2007, 91, 023101.

[74] J. Schütte, R. Bechstein, M. Rohlfing, M. Reichling, A. Kühnle, Phys. Rev. B 2009, 80, 205421

[75] T. Glatzel, L. Zimmerli, S. Koch, S. Kawai, E. Meyer, Appl. Phys. Lett. 2009, 94, 063303.

[76] W. Chen, C. Tegenkamp, H. Pfnür, T. Bredow, Phys. Rev. B 2009, 79, 235419.

[77] M. Fendrich, M. Lange, C. Weiss, T. Kunstmann, R. Möller, J. Appl. Phys. 2009, 105, 094311.

[78] K. Lämmle, T. Trevethan, A. Schwarz, M. Watkins, A. Shluger, R. Wiesendanger, Nano Lett. 2010, 10, 2965.

[79] A. Hinaut, A. Pujol, F. Chaumeton, D. Martrou, A. Gourdon, S. Gauthier, Beilstein J. Nanotechnol. 2012, 3, 221.

[80] L. Nony, F. Bocquet, F. Para, F. Chérioux, E. Duverger, F. Palmino, V. Luzet, C. Loppacher, Beilstein J. Nanotechnol. 2012, 3, 285.

[81] B. Such, T. Trevethan, T. Glatzel, S. Kawai, L. Zimmerli, E. Meyer, A. L. Shluger, C. H. M. Amijs, P. de Mendoza, A. M. Echavarren, ACS Nano 2010, 4, 3429.

[82] A. Hinaut, K. Lekhal, G. Aivazian, S. Bataille, A. Gourdon, D. Martrou, S. Gauthier, J. Phys. Chem. C 2011, 115, 13338.

[83] This value is calculated from the diffusion coefficient of $10^{-7} \mathrm{~cm}^{2} \mathrm{~s}^{-1}$ given in the paper, using the $\operatorname{KBr}(001)$ lattice constant of $6.6 \AA$ and an attempt frequency of $10^{12}$ from applying the formular given in Ref. [92].

[84] P. Rahe, M. Nimmrich, A. Greuling, J. Schütte, I. G. Stará, J. Rybáček, G. Huerta-Angeles, I. Starý, M. Rohlfing, A. Kühnle, J. Phys. Chem. C 2010, 114, 1547.

[85] C. M. Hauke, P. Rahe, M. Nimmrich, J. Schütte, M. Kittelmann, I. G. Stará, I. Starý, J. Rybáček, A. Kühnle, J. Phys. Chem. C 2012, $116,4637$.

[86] R. Pawlak, L. Nony, F. Bocquet, V. Olson, M. Sassi, J. M. Debierre, C. Loppacher, L. Porte, J. Phys. Chem. C 2010, 114, 9290.

[87] M. Kittelmann, P. Rahe, A. Kühnle, J. Phys.: Condens. Matter 2012, 24, 354007.

[88] V. V. Maslyuk, C. Tegenkamp, H. Pfnür, T. Bredow, ChemPhysChem 2006, 7, 1055

[89] V. V. Maslyuk, C. Tegenkamp, H. Pfnür, T. Bredow, J. Phys. Chem. A 2005, 109, 4118

[90] L. Giordano, J. Goniakowski, J. Suzanne, Phys. Rev. Lett. 1998, 81, 1271.

[91] M. Kittelmann, P. Rahe, A. Gourdon, A. Kühnle, ACS Nano 2012, 6, 7406.

[92] R. Gomer, Rep. Prog. Phys. 1990, 53, 917. 